thirds came from the first two classes, and less than one third from the third class. In the same way among those marked fair, 52 of the normal reached this grade against 28 from the deficient. On the other hand, in the lowest grades of scholarship, the defective eyes were the majority. Among 38 marked unsatisfactory, only 4 were normal as to vision, and 15 were from the third class; and in the lowest grade of poor, 42 in all, twice as many had deficient eyes as were normal.

Of course, many allowances must be made in these results for the individual variations in the children in industry and intelligence, but I think these figures strongly suggest that refractive errors and the latent symptoms of eye strain do account for a large part of poor scholarship, and that a large number of poor scholars are probably suffering from defective eyes, though often having good vision. This is a matter of everyday experience to the oculist. Children are constantly being brought who have good enough vision when tested in the ordinary way, but complain of difficulty in doing their school work, and on examination with a mydriatic are found to be suffering with marked degrees of farsightedness or astigmatism, and with proper glasses return to their work without further trouble. Even adults often suffer from marked symptoms of eye strain without being conscious that their eyes are the things chiefly at fault, and this is much more common among children. In this connection it should be understood that the recent act passed by the legislature, ${ }^{1}$ providing for the examination of the vision only of school children by the teachers, while a step in the right direction, will in itself be quite inadequate as a means of finding out all those who are in reality suffering from eye strain.

I found in comparing the results from the lowest to the highest grades that the proportion of the normal children gradually fell off; for instance, in the fourth grade, one half of the children qualified for class one, that is, had normal eyes and complained of no symptoms, while in the highest grade the majority belonged to the second or slightly defective class, and the number in rather urgent need of further examination and treatment equalled the number that were perfect. This coincides with the results of other examinations that have been made among school children, namely, that with advancing education the proportion of normal eyes steadily diminishes and we find an increasing number of nearsighted and astigmatic children. Cohn found in the examination of several thousand children in the schools in Breslau that the normal or model eye was rare, that the farsighted and nearsighted were in the large majority, and that nearsightedness especially tended to increase. In the elementary schools, $6 \%$ were nearsighted, in the Latin schools $26 \%$, and in the university $59 \%$ of all the students. And similar results have been found here, though the average was much better. Among my cases $3 \%$ were nearsighted, and $3 \%$ hat mixed astigmatism.

1 Acts and Resolves of the Legislature of 1906 . An act relative
To sum up my results: Among 420 children, 167 were classed by me as normal, having perfect vision, no ocular symptoms, and no perceptible refractive errors; 155 had little refractive error, good vision and slight symptoms not sufficient to need treatment; 98 had either less than half vision, troublesome symptoms or marked refractive errors, and were mostly referred for further examination.

I found an increasing number of defective eyes in the higher grades, and a majority of normal eyes among the good students, and a majority of defective eyes among the poor students.

\section{AURAL AND NASAL EXAMINATIONS OF SCHOOL CHILDREN.*}

BY D. HAROLD WALKER, M.D., BOSTON,

Assistant Aural Surgeon, Massachusetts Charitable Eye and Ear

THE importance of the examination of the hearing of school children has been recognized in this country only for the last few years, and but recently has it received the consideration it deserves.

In Germany, through the work of Bezold published in 1885 and also that of Kafemann in 1890 , the subject was brought to the attention of the public and since then a great deal of investigation has been carried on.

The importance of perfect breathing, which means full development of the nasal fossa and perfect ventilation of the accessory sinuses and of the middle ear, has been appreciated for some time, but the effect on the scholarship of children suffering from adenoids or nasal obstruction has been more or less overlooked.

In the average public school, there is always a certain number of children who seem inattentive or are considered stupid. There is also a certain number who must be placed in the front row, and every physician who serves in a large aural clinic has seen children who have been sent to the hospital by an observant teacher, who had no ticed that the child watched the teacher's lips or had a discharging and foul-smelling ear.

Considering the above facts, it was thought advisable to make an experiment in order to gather data and, if possible, form some idea as to the best methods for a systematic aural and nasal examination. Therefore the School Board and the Committee on Hygiene in the town of Brookline decided to undertake an experimental examination of the public school children, and for that purpose the Pierce School was chosen.

The children of the upper grade were selected because of their ability to give intelligent answers. The method employed was as follows. The large laboratory was used, the floor being marked in foot spaces up to twenty-five feet. The child was placed in a revolving chair at the first space. The drum membranes, the nose and throat were examined and the condition noted. The hearing was tested by a whispered voice which could be heard by the average normal ear at a distance of 
twenty-five feet, and a spoken voice with thirtyfive feet as the normal limit. The child was placed so that the ear to be tested was toward the examiner, the opposite ear being tightly closed by the forefinger. Numbers from one to one hundred, words and short sentences were used, and the distance at which the child could repeat what it heard, was recorded. Catalogue cards were procured bearing the child's name, grade, etc., having spaces for notes on the examination of the nose and throat and the hearing. In recording the hearing the distance the voice could be heard by a normal ear was used as the denominator and the actual distance the child heard the normal voice, the numerator. As, for an example, a child hearing the voice at fifteen feet, would be recorded as $\frac{1}{2} \frac{5}{5}$, or if heard at five feet, $\frac{5}{25}$.

In the same manner, if the spoken voice were used the denominator would be thirty-five and the distance heard, the numerator. The presence of adenoids was determined by the hearing test, the condition of the drum membranes, whether retracted or not, etc., and the general facial expression. The digital examination and the general use of the mirror were impracticable. The number of children examined was 289 and the results are as follows:

Total number of children having hearing of two-thirds of normal or less $\left(\frac{1}{2} \frac{5}{5}\right), 68$ or $23 \%$; total number having hypertrophied turbinates, 10 ; total number having septal spurs, 35 ; total number having deviation of septum, 8; to.tal number giving evidence of adenoids, 89 or $30 \%$; total number having hypertrophied tonsils, 63 or $21 \%$; total number showing result of chronic suppuration of the middle ear, 15; total number having discharge from ear, 3.

All the children having but two thirds of normal hearing or less, and all those who gave evidence of adenoids and enlarged tonsils were given forms to take home. These requested the parents to take the child to their general practitioner for treatment or for reference to a specialist. It was interesting to note in almost every case of one sided nasal obstruction and deafness, that the degree of deafness was greatest upon that side. This tends to prove the theory that even if there is sufficient ventilation in the naso-pharynx, any nasal contact or obstruction may cause an irritation of the vaso-dilator nerves, causing a congestion of the mucous membrane of the Eustachian tube and the middle ear, which results in a fibrous thickening.

In comparing the hearing tests with the scholarship, it is found that of the pupils marked with the grade of " excellent," $17 \%$ showed diminished hearing. Of those marked " good," $20 \%$ showed diminished hearing. Of those marked " fair," $30 \%$ showed diminished hearing. Of those marked " unsatisfactory," $52 \%$ showed diminished hearing. Of those marked " poor," $42 \%$ showed diminished hearing.

In carrying out these experiments at the Pierce School, itwas interesting to note the great enthusiasm of the principal and the teachers, which seems to have justified the recent appointment of teachers in general, for the examination of the sight and hearing. It is seen by the above results that there exists a very important relation between the scholarship and the hearing and it is to be hoped that parents with children deficient in sight or hearing will appreciate the importance of such deficiencies and will do all in their power to remove them. In cases where parents are careless or decline to provide proper treatment, there should be some provision made to enforce this, in order that the child may be able to take advantage of the opportunities offered by our public schools.

\section{Aqedical Brogregg.}

\section{RECENT PROGRESS IN DIABETES.}

BY ELLIOTY P. JOSLIN, M.D., AND HARRY W. GOODALL, M.D., BOSTON. FERMENTATION AND PHENYLHYDRAZIN TESTS.

None of the tests for sugar in the urine are entirely satisfactory but the fermentation test appears the best for the general practitioner. Pflüger ${ }^{1}$ raises objections to its accuracy, but Salkowski ${ }^{2}$ and Rosenberg ${ }^{3}$ defend it. Salkowski claims that by its use $0.1 \%$ sugar can be surely detected. If the urine is alkaline a crystal of tartaric acid should be added, and the urine quickly boiled and cooled and the yeast then put in. It is a good plan to always add the tartaric acid. It may be necessary to test the yeast from time to time, and the complete fermentation of the sugar should always be determined at the close of the experiment. The ordinary specific gravity bulb has an error of several degrees, so that one only obtains approximate results when the difference in specific gravity is multiplied by the constant 0.23 .

McEwen ${ }^{4}$ has shown that both the typical crystals as well as the thorn apple crystals, obtained by the phenylhydrazin test, can be removed by previous fermentation of the urine with sugar. This test is so accurate that by its use traces of sugar can be demonstrated in practically all urines.

\section{FLUORESCENCE OF DIABETIC URINE.}

Schilling ${ }^{5}$ refers to earlier literature on the fluorescence of the urine in severe diabetes. This appears when $5 \%$ of formalin has been added to the urine and more quickly if a temperature of $50^{\circ}$ to $60^{\circ}$ Centigrade is maintained. He has never found it when the percent of sugar was between $0.5 \%$ and $2.5 \%$, but with urines with $4 \%$ or more. In one case with $5.4 \%$ sugar, it was apparent even after twelve hours at room temperature, and in this case death occurred two months later in coma. The fluorescence gradually increases, and exists unchanged for days and weeks. It is, however, not invariable in urines containing over $4 \%$ of sugar. Schilling was able to show that the fluorescence was not due to sugar, acetone, di-acetic acid or B-oxybutyric 